\title{
Reflections about experiences of compassionate care from award winning undergraduate nurses - What, so what ... now what?
}

Stephen Smith ${ }^{1 *}$, Asha James ${ }^{3}$, Allison Brogan², Elizabeth Adamson ${ }^{1}$ and Mandy Gentleman ${ }^{1}$

\begin{abstract}
Background: From 2007 until 2012 Edinburgh Napier University's School of Nursing Midwifery and Social Care in conjunction with NHS Lothian, collaborated on a programme of action research entitled, the Leadership in Compassionate Care Programme. One strand of this research focused on learning and teaching about compassionate care within the undergraduate curriculum. This debate article focuses on the care issues raised by two award winning nursing students who reflected on the development of their compassionate caring skills during their three year Bachelor of Nursing programme.

Discussion: The reflective accounts debate the following issues related to compassionate care; Personal drivers supporting the provision of compassionate care, Challenging and influencing care practices, Providing relationship centred care and, Living with what can't be achieved. Throughout the debate a model of compassionate care developed from the Leadership in Compassionate Care Programme is used to reflect on key practice issues and provide a framework for practice development.

Conclusion: The care issues presented in this paper identify a need to support students in healthcare to; Develop strategies in questioning care practices which do not meet expectations of compassionate care; undertake focussed reflective activities where each student can explore personal drivers, values and perspectives of compassion; actively connect learning in practice with theory in university, enable development in compassionate caring and strategies that support self-compassion; facilitate an understanding and development of emotional intelligence supporting development of resilience. Implications for healthcare staff are to; Collectively seek, hear and respond to feedback about their service; activate caring conversations within the healthcare team; consider how the model of compassionate care presented in this paper, can be used to reflect on practice and provide a framework for development; consider how we maximise the experience of care during brief moments of contact with patients/families; activate leadership that promotes a culture of openness facilitating the development of compassionate care.
\end{abstract}

Keywords: Reflection, Care, Compassion, Nursing, Relationships, Person-centred, Action research, Undergraduate curriculum, Healthcare students

\footnotetext{
* Correspondence: ste.smith@napier.ac.uk

${ }^{1}$ School of Nursing Midwifery and Social Care, Sighthill Campus Edinburgh

Napier University, Room 4B29, Sighthill Court, Edinburgh EH11 4BN, Scotland

Full list of author information is available at the end of the article
} 


\section{Background}

From 2007 until 2012 Edinburgh Napier University's School of Nursing Midwifery and Social Care in conjunction with NHS Lothian, collaborated on a programme entitled, the Leadership in Compassionate Care Programme (LCCP) [1]. NHS Lothian provides a comprehensive range of primary, community-based and acute hospital services for the second largest residential population in Scotland circa 800,000 people, it employs approximately 24,000 staff. The School of Nursing, Midwifery and Social Care at Edinburgh Napier University provides undergraduate and post graduate education to nurses, midwives and multidisciplinary staff involved in health and social care. There are approximately 1600 students in the school.

The LCCP utilised an action research approach with the aim of embedding compassionate care in NHS healthcare practice and within undergraduate nursing and midwifery education. The LCCP encompassed four key strands of work: developing compassionate clinical practice; supporting newly qualified practitioners; leadership development and undergraduate nurse education. This action research programme was funded by a private benefactor. A key outcome of this action research identified a model for compassionate care in practice which incorporates six components, see Fig. 1. This model relates to activities undertaken between care providers, service users and their families/important others, for example caring conversations should take place between care providers as well as between staff and service users. Debating, challenging and celebrating care provision is an important activity to be undertaken amongst staff. The LCCP action research identified that this was a key activity if compassionate care is to flourish in a care setting. Throughout this article reference will be made to this model and how the reflections presented highlight its key components.

This debate article focuses on the care issues raised by two nursing students (adult field) who reflected on development of compassionate caring skills during their three year Bachelor of Nursing programme. These reflections were written as part of their successful submission for the Simon Pullin award. The Simon Pullin award is given to students who have shown excellence in the development of person centred, compassionate caring skills during their programmes of study. The award is dedicated to the memory of Simon Pullin, who was a Senior Nurse on the Leadership in Compassionate Care Programme from its commencement in December 2007 until his death in July 2011. This award highlights the fundamental importance of compassionate care as part of contemporary Nursing and Midwifery practice. The award winners Alison Brogan and Asha James have contributed their reflections and analysis of compassionate care in the planning and writing of this paper and they have agreed to be identified within this writing. The key issues related to the provision of compassionate nursing care debated in this article are as follows:

1. Personal drivers supporting the provision of compassionate care

2. Challenging and influencing care practices

3. Providing relationship centred care

4. Living with what can't be achieved

This article incorporates the What, So what and Now what [2] reflective model as a way of reflecting on experiences of compassionate care.

Borton's model asks individual's to consider three questions: "What?", "So what?" and "Now what?" The first question asks a person to consider what has happened, the second question is to allow the person to try and make sense of what has happened and evaluate events and the last question is to consider a way forward and think about what could happen in the future [3].

The LCCP identified that discussions about care 'caring conversations' and working with feedback are key activities enabling compassionate care to thrive. The use of a reflective model acts as a as a springboard supporting such discussions in practice (Table 1).

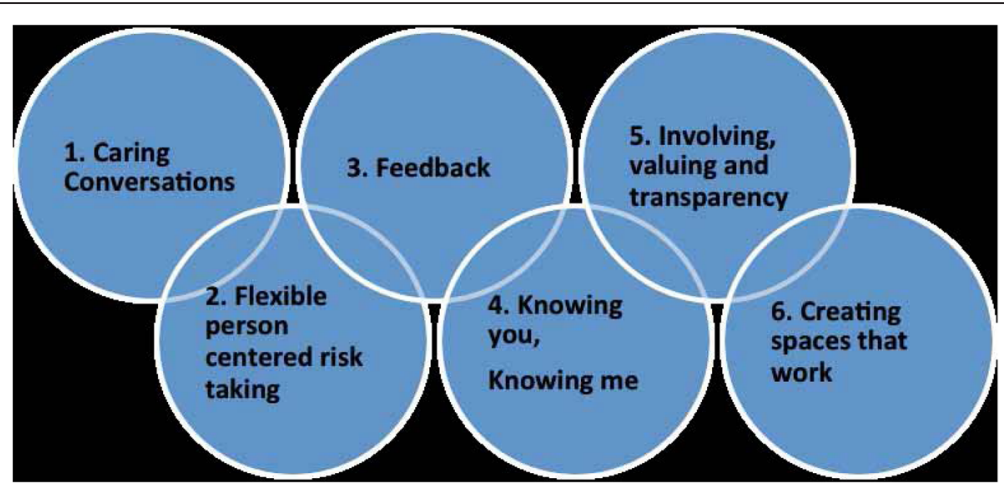

Fig. 1 Model for compassionate care in practice [14] 
Table 1 Working with Borton's reflective model

\begin{tabular}{|c|c|c|}
\hline What? & So What? & Now What? \\
\hline $\begin{array}{l}\text { This is the description and self- } \\
\text { awareness level and all questions } \\
\text { start with the word "what". } \\
\text { This stage combines the reflective } \\
\text { processes of identifying the } \\
\text { experience and describing it in } \\
\text { detail. }\end{array}$ & $\begin{array}{l}\text { This is the level of analysis and evaluation when we } \\
\text { look deeper at what was behind the experience. } \\
\text { This stage breaks down the situation and tries to } \\
\text { make sense of it by analysis and evaluation, drawing } \\
\text { on previous experience and knowledge. }\end{array}$ & $\begin{array}{l}\text { This is the level of synthesis. Here we build on the } \\
\text { previous levels these questions to enable us to } \\
\text { consider alternative courses of action and choose what } \\
\text { we are going to do next. } \\
\text { This stage combines the process of exploring } \\
\text { alternatives and planning actions that will be put into } \\
\text { practice. }\end{array}$ \\
\hline $\begin{array}{l}\text { Examples } \\
\text { What happened? } \\
\text { What did I do? } \\
\text { What did others do? } \\
\text { What was I trying to achieve? } \\
\text { What was good or bad about the } \\
\text { experiences }\end{array}$ & $\begin{array}{l}\text { Examples } \\
\text { So what is the importance of this? } \\
\text { So what more do I need to know about this? } \\
\text { So what have I learnt about this? }\end{array}$ & $\begin{array}{l}\text { Examples } \\
\text { Now what could I do? } \\
\text { Now what do I need to do? } \\
\text { Now what might I do? } \\
\text { Now what might be the consequences of this action? }\end{array}$ \\
\hline
\end{tabular}

\section{Discussion}

\section{Personal drivers supporting the provision of compassionate care}

I was a child carer from a very young age for my mum, who suffered with alcohol dependency. Life was far from easy, but I always understood that my mum did not choose to live like this and what she had was an illness. However it appeared that many healthcare professionals did not share this understanding and their lack of compassion, respect and dignity often left my mum feeling judged, embarrassed and worthless. This caused a lot of distress for my mum and for the family. This was the beginning of my dream of becoming a nurse... if I was a nurse I would be kind to my patients and care for everyone the way I would have liked my mum to be cared for.... The negative attitudes we experienced as a family by healthcare professionals have enforced my passion for person centred, compassionate care.' Asha

It is evident from this reflection that past negative experiences of healthcare had provided a strong personal driver to enter the nursing profession but moreover to develop a future focus on care that is compassionate and directed towards the person, their needs and their context. The negative elements of this account point to feelings of injustice, frustration and are a consequence of care experiences resulting in emotional distress for a vulnerable person and their family. It is indeed commendable on a personal level that despite these significant negative experiences this generated a desire for Asha to become a nurse and ultimately lead to successful completion of a nursing degree and recognition of excellence in the development of her compassionate caring skills.

This reflection triggers questions about how health and social care educators can support students to learn from their life experiences and consider their individual values and how this may support developments of caring and compassionate attributes in practice. In Asha's reflective account, development in compassionate caring arose from her previous negative experiences of healthcare and this appears to have been integral to her approach to care and her values. It is necessary to support each student within their own context and personal experience. When considering Asha's experience of caring for her mother, the complexity of this situation and the impact on Asha's own health and wellbeing are key factors to consider.

'Like many children in my situation, I was exposed to domestic violence and parental mental health illness, as a result my functioning was adversely affected. School in general was a struggle and I left with practically no qualifications. I had no confidence and low self-esteem. When I was twenty four, my mum died, she had Cirrhosis of the liver. This was a devastating time for myself and my family. I went to counselling and gradually started to turn my life around. It's taken many years and with the support of my husband, who has always believed in me, I started college to gain the qualifications I needed to get me into nursing. I could not ask for more. I am confident and happy in what I am doing today.' Asha

It is clear from this candid reflection that considerable work and energy from Asha herself, her family and professionals have enabled a sense of wellbeing and healing to emerge. This in turn enables her to practice with emotional intelligence and to raise a challenge with colleagues when care standards are not achieved. It is critical to consider how colleagues, teams and organisations support this maintenance of functioning over time.

Questions are therefore raised as to how we support learners who have less clear articulation of past experiences of care, their personal values and where this leads in the development of caring skills. Indeed how does this issue play out for learners who have directly experienced 
abuse or life contexts where experiences of compassion would be deemed less than positive? There appears something fundamentally crucial about mirroring a compassionate, person centred learning experience for our students whilst we encourage them to explore their values and approach to care. This in turn raises questions about academic staff modelling attributes of care and compassion within their professional practice.

As we have discussed when students embark on their programme of study they bring with them their own life experience, values and beliefs, and some of these will be challenged as they experience healthcare practice. One way that students can be encouraged to reflect on the experiences of those who give and receive care is through listening to and reflecting on stories gathered within clinical practice and relating this to their own experiences of giving care $([4-6,1])$. Nursing students often make reference to their own personal care encounters, episodes that have been deemed less than compassionate can present a strong driver for student nurses to achieve more positive experiences for others. Teasing out learning from stories provides a student centred approach to considering issues of care and compassion. What do I make of the care experience? What would I have done in this situation and why would I respond in this way? What possibilities of compassionate caring are evident in this story/context? Reviewing stories in a group learning context facilitates the student to further explore varied perceptions and approaches to care and compassion. This form of group learning requires careful facilitation enabling students to safely question practice and approaches to care, then consider possibilities for their future practice.

The intertwining of practice learning experiences with the students' life experiences and values provides a potentially provocative opportunity for development. There are some thorny practical issues here that require deliberation if we are to maximise this practice learning. How do we support students and mentors to capture these learning stories from practice and how do we integrate these within theoretical learning about compassionate care within the university context? If personal stories and narratives are a key component of learning about compassionate care we need to activate the integration of this learning between theory and practice.

Caring is fundamental to nursing and therefore must be an integral part of nurse education. Concerns about poor care and lack of compassion are frequently reported in the media, those responsible for nurse education are acutely aware of this as they develop curricula and focus on preparing students for the profession. Whether compassionate care can actually be taught has been debated [7]. McLean [8] makes a case for a values based curriculum that encourages self-awareness through a values based enquiry model which encourages the student to challenge existing beliefs and values and develop the character they require to be providers of care and compassion. Nurse Educators can teach the theory of compassionate care but application in practice can be challenging as demonstrated in the reflective accounts in this paper. Curtis [9] found that student nurses wanted to provide compassionate care but felt unsure of their capacity to do this and sustain it in practice. Others suggest that students' ideals of care, though already present as a strong motivator when they commence their studies, increased as they grew in knowledge and competence [10]. They also found that when students experience moral distress when these ideals are challenged through witnessing poor care, these ideals are upheld. These emotionally challenging situations can however become a vehicle for personal and professional growth when students find the courage to uphold their ideals and increase their ability to provide care that is more compassionate [10].

While nurses are focused on caring for others, it has been argued that practitioners require to be compassionate with themselves [11], and this is associated with the development of emotional intelligence [12]. The emotionally intelligent nurse is said to be one" that can work in harmony with thoughts and feelings" ([13], p. 94) therefore is able to respond to both when working directly with patients and their families. This form of emotional intelligence provides the potential of understanding and connecting with those being cared for whilst recognising and responding to personal strengths and vulnerabilities. Research evidence suggest a connection between emotional intelligence and cultural intelligence (Moon, [14]) and this is evident in the reflections of care shared by Asha and Allison. Cultural intelligence relates a person's capacity to function successfully in culturally diverse situations (Ang, Dyne \& Koh [15]). In the account of a clinical placement experience overseas, Asha expressed astonishment and concern at the discriminatory custom and practice she encountered. The cultural approach to care for indigenous people was in direct conflict with her ideals of compassionate person centred care and she felt unable to act in the way directed. Instead she engaged in building a trusting relationship with the family and was rewarded with expressions of gratitude. Not only did it take courage as a student to challenge the practice of a registered nurse but particular resolve and determination to do this in a different culture. By demonstrating how a person centred accepting approach could be not only successful but welcome by a patient and their family she challenged her colleague.

Whether compassionate care can actually be taught has been debated [7]. McLean [8] makes a case for a values based curriculum that encourages self-awareness 
through a values based enquiry model which encourages the student to challenge existing beliefs and values and develop the character they require to be providers of care and compassion.

In addition it has been argued that unless a healthcare practitioner is compassionate to themselves they cannot provide compassionate care for others [11], and this is associated with the development of emotional intelligence [12]. The emotionally intelligent nurse is said to be one" that can work in harmony with thoughts and feelings" ([13], p. 94) therefore is able to respond to both when working directly with patients and their families. This form of emotional intelligence provides the potential of understanding and connecting with those being cared for whilst recognising and responding to personal strengths and vulnerabilities. Research evidence suggest a connection between emotional intelligence and cultural intelligence (Moon, [14]) and this is evident in the reflections of care shared by these students.

Considering the model of compassionate care identified by the LCCP [1] it would seem appropriate that Caring conversations and Feedback would be critical components of the model to concentrate on. Observing and participating in caring conversations in practice will provide opportunities to hear debates and have discussions about how to care, and develop understanding of the real dilemmas of care. In addition receiving feedback about their own caring and compassionate skills from practice mentors will complement the potential for students to develop caring skills and reflexive practice. All of these activities will provide opportunities to both challenge and construct learning based on their own drivers and values.

\section{Challenging and influencing care practices}

Both of our students incorporated the issue of challenging and influencing compassionate care practices within their reflective accounts. They took different approaches to influence staff. It is recognised that for students and NHS staff raising concerns about practice with mentors and practice staff can be a daunting experience [16].

'Encouragingly I recognised that as a third year student I was starting to feel confident in being able to influence colleagues. Whilst in the community setting I cared for a patient who could be rude and verbally aggressive. He was abrupt with me during my first solo visit and referred to me as student. He had COPD and leg wounds, he was very particular about everything and was quite obsessive. I realised that this man had little control over anything in his life and I suspected this is what drove his behaviour. By my third visit I had worked out exactly how he liked things done and instead of letting him get anxious and out of breath telling me what to do, I gently took control of the situation. I did things in the order he liked..... this didn't take any more time - in fact it saved time. By the end of my fourth visit he asked my name. I was able to relay this during handover with my community colleagues and I explained that I thought his behaviour had been driven by fear and loss of control. With this understanding and consideration of what was driving his aggressive behaviour his relationships with the nursing team improved significantly. He seemed more content during nursing visits and so did some of the nursing staff.' Allison

This reflective account highlights a particular approach to a challenging caring situation, namely approaching it with eyes wide open, thinking what is happening here and what could my contribution be? It is evident behaviours were observed with a view to understanding them and importantly considering how this guided practice. It is evident that emotional intelligence was key in considering and moderating practice behaviour in this context. 'Theoretical and editorial literature confirms emotional intelligence concepts are central to nursing practice. Emotional intelligence needs to be explicit within nursing education as emotional intelligence might impact the quality of student learning, ethical decision-making, critical thinking, evidence and knowledge use in practice' [17]. The challenge to her community colleagues was to adapt their practice in the light of an enhanced understanding of the person's situation. Talking about this openly with colleagues as part of a formal forum facilitated an enhanced change of communication style and active recognition of the reasons behind the patient's irritable behaviour. In turn this gave an opportunity to consider action/practices which would enhance the care experience for both the patient and the nursing staff? Smith et al [18] highlighted the importance of reflective forums when considering an understanding of compassionate care in the workplace, findings from this action research identified three key themes; leadership, culture, professional and personal development. It is evident that these findings and key themes have congruence with Alison's reflective account and the outcomes for the community team.

The influence and challenge here was based on Alison's perceptions and experience of care provision. She used her own observations and positive practice experience to influence colleagues asking, can we learn from what I have done? This approach differs to asking the question what can we do about this? This way of influencing appeared natural and real, focusing on an approach which had been successful in practice ensured relevance for staff and additionally provided tangible possibilities for development. 
Allison also identified,

'The nurse manager in this setting was a role model to the team and often openly reflected on her experiences both positive and negative. This encouraged the nursing team to mirror this practice.... The team got together twice daily to discuss patient care. The nurse manager would often make a point of asking what went well and what would you improve. Change was not something to be feared rather it was embraced.'

With this insight Alison was able to utilise this team's strengths to influence a development in care, further evidence of active emotional intelligence impacting upon practice.

Asha's influence on care practices adopted a different approach.

I was fortunate to undertake a student placement in Western Australia, I was placed in a medical ward in one of Australia's public hospitals where many of the patients lived in relative poverty. This was a fantastic learning opportunity for me..... There were a lot of aboriginal people attending the hospital and I witnessed some discrimination from Australian healthcare professionals which really surprised me. I was given three patients to care for, one was aboriginal. The nurse I was working with said to me that when caring for this particular patient I had to leave the room once I had done what I was supposed to do. I was not to make eye contact with the patient or the family and not to engage in conversation other than health related topics. I could not believe that I was hearing this from a healthcare professional, this goes against my own values and everything I have been taught. I challenged the nurse about her behaviour..... I was told that this is just how it is and aboriginal people take it as a threat if you make eye contact. I explained I cannot provide care like this and thought it was only fair to treat people as individuals. The next time we worked together she was surprised at how quickly I had built good trusting relationships with the patient and family. I spent time demonstrating empathy, kindness, dignity and respect I got to know everyone..... The family expressed how grateful they were to me for my care which made it all worthwhile!' Asha

In this reflection the challenge to practice was forthright and highlighted concerns about the provision of compassionate, person centred care. It would appear that a growing awareness of discriminatory care in this clinical context triggered this response to the mentor. It is evident that Asha demonstrated commitment to the quality of care she wanted to provide, moreover she was able to articulate this clearly and challenge current practice with her mentor. This confidence is admirable given she was working in an unfamiliar setting where a culture of discrimination had been identified and working with unfamiliar staff. This brief scenario demonstrates a student nurse whose values, life experience, academic development and clinical practice enabled this way of challenging practice. Clinical confidence, commitment to quality of care and influence is demonstrated and indicates that this can be achieved by student nurses towards the conclusion of their studies.

Asha stated,

'when you are a student it is quite easy to feel unsure of yourself or your skills as a nurse, especially when faced with a new situation. Challenging my mentor was not something that came easy to me, however, being firm in my belief by speaking up I feel certain I have helped and cared. I continually set goals to push myself to have the confidence to speak up if I felt it was important to me. You are always going to meet people in life with negative attitudes, but it is important to me not to let them influence my thoughts and actions in any way. I found it difficult relating to the nurse who I reacted to as I felt that she stereotyped this patient and her family. I have no doubt that my own personal experience motivated my decision to question her practice.

Having a bit of knowledge also gave me confidence to speak up. I had done a little research about communicating with Aboriginal people prior to visiting Australia, and I was aware that some (but not all) Aboriginal people are uncomfortable with direct eye contact. I felt that I was mature enough to make my own judgements on how to communicate effectively to overcome any barriers.'

Within recent public failures of NHS care, an inability to speak up and raise concerns about practice were highlighted as a factor in the general lowering of care standards, recommendations aimed at addressing this culture and behaviour were identified, 'Ensure openness, transparency and candour throughout the system about matters of concern' [19]. It is therefore critical that caring conversations are healthy and can positively influence the behaviour and care provided by practitioners and the culture of the caring environment. There is evidence of a need to talk about our healthcare organisations with positive values and a clear vision of how compassionate organisation should function [20].

It is evident that feedback from the patient, their family and the mentor indicated that the care experience provided by Asha was very positive. Unfortunately from 
this brief reflection we do not have evidence of influence on the mentors practice in this area of care other than her surprise at the positive level of therapeutic relationship developed. There is evidence however that the care provided to this patient and family was influenced and became consistent with Asha's standards of compassion and person centeredness.

As discussed above Asha made earlier reference within her reflective account to personal experience early in her own life where she experienced discrimination as a consequence of family circumstances, and which enabled her to empathise with the indigenous family who may have felt isolated and misunderstood. Determination to provide compassionate care and challenge established practice took courage, confidence and commitment all of which are identified as fundamental values of care [21]. This emphasises the need to actively engage with each student throughout their period of study exploring their values, attitudes and caring behaviours.

Cultural sensitively is clearly an element of compassionate person centred care as demonstrated in this example and there is growing awareness that this should be taught within nursing education [22]. For some time nursing curricula has focused on fitness for practice and clinical competence and this is vital for patient safety but nursing is more than this. Nurses education is increasingly challenged to ensure that graduates are equipped with a spectrum of knowledge, skills and ability where compassionate, person centred care is consistent with safe and effective care. This requires acknowledgement that the students who enter our programmes of study come from a variety of life experiences and cultural backgrounds and as educators we too must meet the challenge to provide a learning experience that addresses and meets the needs of a diverse group of students. This raises the question of how this can be done and how can we ensure that nursing graduates are ready to meet the needs of the people in their care, can also care for themselves, and be able to grow with an ever changing healthcare system?

In relation to the LCCP model of compassionate care the examples provided above in relation to challenging and influencing care highlight the themes; Person centred risk taking and Involving valuing and transparency. Both reflections highlight practices that were focussed on adapting practice to individual need, for example following the instructions of the patient in order to develop trust and reduce anxiety whilst understanding their need for control. Similarly having an awareness of cultural practices but focusing and responding to the patient as an individual. Risks taken here related to deliberately doing things differently from colleagues and experiencing potential negativity as a consequence. Also taking a different approach may not be accepted by colleagues in the long run.

\section{Providing relationship centred care}

II will never forget one of the patients I cared for. After ten weeks she shared that she had outlived all her relatives ..... she said she didn't know what she had done to deserve this and was so lonely she had no one to cuddle her, then she asked me for a hug. I will never forget the solitary tear drop running down her face when she gave me a hug. I wonder how long it had been since that woman had been hugged? This is one defining moment of my training which makes me strive to build appropriate compassionate therapeutic relationships at every opportunity.' Allison

This example from practice highlights that personal connection is important in the delivery of compassionate care and that although the patient's overall care was not being criticised, the emotional aspect of her being had somehow been forgotten. The question, what was important to that individual at that particular time had not been asked. This example also shows that relationships can take time to foster and develop. After 10 weeks of contact with Alison, this woman shared her feelings and vulnerability allowing Alison to respond to her needs in a simple but profound way. There is much debate in the nursing literature focused on terms such as relationship centred care, person centred care and compassionate care [23]. Following a pragmatic stance they each point to an approach to care that focuses on enhancing the experience of care itself, however relationship centred care provides a balance to this approach whereby the experience of the care giver and care receiver must equally be acknowledged to achieve an overall enriched environment of care [24].

This care experience emphasises the importance of touch, specifically the request for a hug when feeling isolated and lonely. Perhaps focusing on the appropriate use of touch within a caring relationship can support staff to maximise the caring experience when brief moments are all that are available to staff within busy clinical settings. Touch is not comforting or appropriate for everyone but the questions is, how can we maximise the appropriate use and effectiveness of touch when we have minimal time to care?
'Many nurses hold the opinion that building relationships and honing communication skills are key to excellent patient care.... During my first year at university I observed that building therapeutic relationships and adapting communication style both saved time and improved patient care. I observed a colleague waking a patient abruptly in keeping with ward routine. The patient who had dementia did not take kindly to being rushed out of bed. She became 
tearful and ultimately aggressive. This resulted in two members of staff having to assist in calming the situation. In contrast, I witnessed another nurse gently rouse the patient in the morning. She held her hand and physically brought herself down to her level. This nurses' tone and demeanour was gentle and patient centred, this initial interaction did take longer, however overall it saved time as the patient was happy to allow staff to assist her with her daily care. Inconsistency within the nursing team on this particular ward was very evident.' Allison

How do we share and provide an opportunity to maximise good practice such as described in this account, and what is the role of a leader in promoting and sustaining compassionate care such as this? Role modelling has been celebrated in an earlier reflection by Allison. The intent and behaviours of leaders are important when establishing a culture of openness and transparency where feedback is viewed as "the norm". This form of culture facilitates the possibility of sharing and maximising positive practice as well as giving consideration to practice situations which are challenging and fraught. In essence this is working directly with local feedback from patients and staff and holding a persistent focus on caring conversations.

'What factors drive poor behaviours like this where there appears to be inconsistent approaches to patient care? Notably, the nurse manager on this ward was rarely available. The lack of direct leadership resulted in poor team work and communication within the team. How can we create environments where all patients are given this type of individualised care? Is this primarily driven by influential leadership? I believe a strong inspirational leadership style is a key factor in driving culture change? I believe cultures can be changed when staff are empowered to suggest improvement and champion change.'Allison

This reflective account highlights the key theme of 'Creating spaces that work'. What would it take to develop consistency of practice within the care team? Allison's focus rests on leadership but moreover the need for the team to share, debate and consider how care can be enhanced, in essence work with 'Caring conversations'.

It is considered that most nurses will experience what is commonly termed Compassion fatigue. This concept has been debated widely within the nursing arena. Coetzee and Klopper [25] conclude that this this develops gradually and is a cumulative process. It is thought that this occurs when one is consistently subject to intense, acute interactions with patients and families. Compassion fatigue can be described as feeling negative emotions as a result of feeling unable to fulfil their moral role as nurses. Have more experienced healthcare providers become burnt out? In tandem with compassionate skills should we should also teach nurses to avoid becoming fatigued. This would allow compassion to be preserved rather than eroded. If this is the case how do we go about safeguarding ourselves and others from the effects of compassion fatigue? Indeed, what makes one person more adept at sustaining their compassion whilst working in highly charged emotionally draining environments? Can resilience be taught or nurtured? Gentry [26] highlighted that a healthcare providers ability to "self-care" seemed to have a positive influence on their ability to deal with the effects of compassion fatigue.

Self-care was identified primarily as anxiety management and included practices such as speaking to colleagues, exercise and meditation. During the three year nursing programme at Edinburgh Napier University students are given ample opportunity to reflect in peer groups there are also sessions teaching mindfulness. During one module nursing students were invited to organise aerobic exercise classes. Is the UK nursing workplace ready or able to uphold and commit to providing organisational strategies to combat the effects of compassion fatigue? There has been little research into techniques on how to reduce fatigue in healthcare professionals and the resulting effect this would have on standards of care, this is an area which would benefit from further research.

'Within one clinical setting where I worked a colleague experienced the loss of her spouse. She was regularly involved with palliative patients and was given the option to move to a different role within the team. This role did not involve palliative care and is allowing an experienced and dedicated member of staff to better deal with her grief. It also means she is still effectively contributing to the workplace. What makes it possible for one clinical area to offer this type of support where as other areas do not? How can this become the norm as opposed to the exception?' Allison

The example of good practice above highlights that relationship centred care is critical to staff relationships. This provides a practical but considered example of the theme Knowing you knowing me. Understanding a staff members context and vulnerability and responding to this may enhance the ability to care in practice and sends a message to staff that they are of value and matter.

As a student nurse, the relationship between myself the patient and their family is vital. Holistic care and having excellent verbal and non-verbal communication skills are essential in order to understand the needs of 
each patient and their loved ones whilst they are receiving healthcare... I have learned how a person's body language can say a completely different thing as to what they are telling you, thereby taking the time to listen and by doing the little things can not only make the patient feel cared for but actually cared about.' Asha

The authors of this paper would acknowledge that courage can be required to pick up on non-verbal behaviours and then comment or respond to them [21]. These forms of interaction may take longer than originally anticipated but aim to get at the heart of what matters. In addition recognising the needs of the family/carers in the provision of care to promote a sense of caring for all involved is also important.

\section{Living with what can't be achieved}

'For the first time I felt inadequate in terms of providing compassionate care. It was my first exposure to an emergency medicine environment. During one shift I was very conscious of a daughter sitting with her mother. The mother was having an acute breathlessness problem and her daughter was crying. I was dealing with a patient who had a gastric bleed. On my way to get some clean linen for this patient, the only thing I could offer this distressed daughter was a tissue and a few words which seemed very inadequate... I really felt I was letting someone down.... My thoughts were interrupted when my patient said you are so gentle thank you for being so gentle. I realised I was doing my best and providing care to the patient with the greatest need.... The lesson learned here is that in the demanding role of nursing I will have to prioritise and make decisions such as these.' Allison

This reflection highlights the dilemma experienced by care providers when they identify distress and are unable to respond in a manner that meets their aspirations of care. A feeling of inadequacy related to compassionate care provision was acknowledged, this did not relate to an absence of compassion or a failure to respond, rather it points to a busy clinical context where priorities need to lie with those who have the most acute needs. A compassionate response was evident in the provision of a tissue and a few words and this is commendable practice amidst a busy clinical setting. It is interesting to note however that this intervention was deemed inadequate.

This reflection raises a number of key questions about the context and environment of care and the impact these factors have upon staff experience.

Was this area short staffed or experiencing an acutely busy period and feeling stretched at this time? What is the norm of practice here, do other members of the team feel similarly and where are these experiences and feelings discussed and debated as a team?

How would other members of the team manage this situation and how can staff retain their awareness and desire to be compassionate when the busyness of the area necessitates a focus on clinical priorities?

A further question relates to the resilience of staff, how do staff stay resilient and work with potential compromises to the compassionate care they aspire to deliver?

Brown [27] debates these issues concluding that, 'in organisations there needs to be clear intention, leadership and determination for compassionate care to become central in all healthcare practice'. His conclusions focus on small group experiential reflection and learning by healthcare workers as the most effective way to consolidate compassionate care values in practice. Indeed within the LCCP [1] staff coming together to debate and discuss what compassionate care looks like in their service was a key activity in negotiating a definition for practice; moving the potentially nebulous concept of compassionate care into a more tangible approach for local practice. Principles of compassionate care were most usefully derived from hearing and understanding experiences of patients, relatives and staff and initiating responsive action [28].

Paley [29] argues that organisational and situational factors directly impact upon care delivery and that this results in care providers being too busy or focused on other organisationally directed issues to recognise or address compassionate care. It is argued that the blame for a compassion deficit therefore rests with the organisation. In a related issue Timmins and De Vries [30] also point to organisational factors that determine outcomes of poor care. These authors focus on cognitive dissonance as experienced by care providers; the aim is to provide good care however due to organisational pressures which dominate the context this form of care is not achievable. In order to rationalise this dissonance between care aspirations and the actual, less positive, care experience, standards of care are revised to a lower level thereby managing the dissonance experienced. In the reflection provided above neither of these two phenomenon were evident. Frustration was expressed due to the busyness of the practice area and a need to focus on clinical priorities but distress was identified and a compassionate response elicited.

It is interesting however that this student was dissatisfied with her compassionate response in this busy context, this response raises questions about how staff are supported and able to work through these situations when optimal care has not been possible. There is a wealth of evidence available testifying to the significant challenges facing healthcare professionals in contemporary practice (Iles, \& Vaughan Smith [31] therefore it is 
important to consider how staff develop resilience and self-compassion as a counter to this situational and cultural context as well as responding to physical and organisational factors impacting upon compassionate caring.

The following practice example from Allison demonstrates tensions in achieving her desired standards of care and how she has been developing strategies to address these.

'I have been a qualified nurse for a year now and often miss being a student. There was certainly more time to spend with patients. Instead of supporting an upset patient with post-operative nausea and vomiting it is my first priority to organise the administration of an anti-emetic. There is often no extra time to spend with the patient following this as I could have another patient returning from theatre. How do we make these shortened interactions count and make our patients feel cared for as an individual? Is it about tone and communication style? Is it about delegation and recognising that other members of the team can help. I have certainly asked student nurses or care assistants to comfort patients when I have been unable to fulfil this role due to time restraints.' Allison

The challenge raised of maximising the positive impact for patients of brief interactions when a brief moment is all that is available, seems an important focus for learning. It is unreasonable for staff to experience a sense of guilt regarding care experiences such as those described above when staffing and situational variables dominate practice and are out with a nurses' sphere of control or influence. What would help nurses and other care staff discuss their experiences, learning, and the positive strategies employed which would enable the possibility of working towards a shared understanding and improvement?

In regard to the LCCP model of care the key themes related to the reflections above could relate to Feedback and Creating spaces that work. It would have been fascinating to hear directly from the patients described in the reflections detailed above. What was their perception of the care and compassion they received? Did a tissue and a few words, or the provision of an anti-emetic meet their needs? Patients can be acutely aware of the busyness of clinical areas and consequently try to minimize the work load of staff as a response. Without seeking feedback directly from these people and their experience of care, we are left with assumptions and possibilities. There is the possibility that care staff can be unduly critical of their practice when deficits are perceived in the care provided, however such deficits may or may not be the experience of those we care for. Actively seeking, hearing and responding to feedback within the local context can provide a reality check and focus for both celebrating excellent care and genuinely understanding care deficits. Considering, Creating spaces that work, would focus reflections on issues connected to the physical environment, culture and ways of working. A group discussion may reassure the team that they are doing all they can, it may raise possibilities for change or connecting with the broader organisation for direct support and development.

\section{Summary - Now what?}

The student nurse reflections discussed within this paper prompt us to pause and be encouraged as we glimpse examples of excellent care and celebrate what can be achieved. They also remind us that provision of compassionate care is indeed possible, it does take place on a day to day basis and often hinges on the little things that make a massive difference to those receiving and providing care. However personal drivers, values and perspectives of quality care are critical aspects of providing compassionate care. These elements though are not little things to articulate and develop for individuals, teams and indeed organisations engaged in the provision of contemporary healthcare.

To support the development of compassionate care this article focused on key issues identified from the reflective accounts of two award winning student nurses. These included supporting students in healthcare to;

- Develop strategies in questioning care practices which do not meet their expectation of compassionate care

- Undertake regular focussed reflective activities where each student can explore personal drivers, values and perspectives of compassionate caring.

- Actively connect learning in practice with theory and reflective activities within university, enabling consistent development in compassionate caring.

- Facilitate an understanding of emotional intelligence, leading to strategies for self-compassion and the development of resilience

It is necessary to consider the implications of these reflections in terms of supporting healthcare staff to;

- Collectively seek, hear and respond to feedback about their service

- Activate caring conversations within the healthcare team, celebrate what works well and understand the reasons for this, considering what can be done differently and better and how can we learn and support one another.

- Consider how the model of compassionate care, presented in this paper, can be used to reflect on 
practice and indeed provide a framework and focus for local practice development.

- Consider how we maximise the experience of care during brief moments of contact with patients/ families.

- Activate leadership that strives for a culture of openness which facilitates the development of compassionate care.

\section{Abbreviations}

LCCP, Leadership in Compassionate Care Programme

\section{Acknowledgements}

None.

\section{Funding}

This paper does not present material from funded research.

\section{Availability of data and materials}

The data on which the conclusions of the manuscript rely are presented in the main paper.

\section{Authors' contributions}

All authors have contributed to the writing of this paper in accordance with the International Committee of Medical Journal Editors (ICMJE) guidelines.

\section{Competing interests}

There are no competing interests identified related to the writing and publication of this paper.

\section{Consent for publication}

Consent has been sought from participants whose accounts have been included in this paper.

\section{Ethics approval and consent to participate}

The information presented in this debate article does not originate from original research but from material presented by undergraduate nursing students as part of an award portfolio submission. As this paper does not represent research no ethical approval was sought.

\section{Disclosures}

Students who win the Simon Pullin Award receive $£ 250.00$ prize money and an award letter from the university. The application process involves submitting a portfolio which contains the following: a reflective account written by the student identifying their experience of learning and development in compassionate caring during their undergraduate studies; accounts of feedback about compassionate care development from practice mentors, service users and university academic staff, usually the students' personal development tutor. The portfolios are reviewed by a panel of academic staff and service users.

\section{Author details}

${ }^{1}$ School of Nursing Midwifery and Social Care, Sighthill Campus Edinburgh Napier University, Room 4B29, Sighthill Court, Edinburgh EH11 4BN, Scotland. ${ }^{2}$ Distict Nursing Team, NHS Lothian, Edinburgh, Scotland. ${ }^{3}$ St John of God Hospital, Perth, Western Australia, Australia.

Received: 9 January 2016 Accepted: 1 June 2016

Published online: 11 July 2016

\section{References}

1. Edinburgh Napier and NHS Lothian. Leadership in Compassionate Care Programme, Final Report. Edinburgh: Edinburgh Napier University; 2012.

2. Borton T. Reach, Touch and Teach. London: Hutchinson; 1970.

3. Jasper, M. Beginning reflective practice. Nelson Thorne: Cheltenham; 2003.

4. Adamson E, Dewar B. Compassionate Care: Student nurses' learning through reflection and the use of story. Nurse Educ Pract. 2015;15(3):155-61.

5. Adamson E, Dewar B. Compassion in the nursing curriculum: making it more explicit. J Holist Healthc. 2011;8(3):42-5.
6. Adamson E, Smith S (2014) Can Compassionate Care be Taught? Experiences from the Leadership in Compassionate Care Programme, Edinburgh Napier University \& NHS Lothian IN Shea, S., Wynyard, R., Lionis, C. (2015) Providing compassionate healthcare: challenges in policy and practice. Routledge Oxon.

7. Bradshaw A. Measuring nursing care and compassion: the McDonaldised nurse? J Med Ethics. 2009;35:465-8.

8. Mclean C. The yellow brick road: a values based curriculum model. Nurse Educ Pract. 2012;12(3):159-63.

9. Curtis K, Horton K, Smith P. Student nurse socialisation in compassionate practice: a grounded theory study. Nurse Educ Today. 2012;32(7):790-5.

10. Pedersen, Birgith, and Kerstin Sivonen. "The impact of clinical encounters on student nurses' ethical caring." Nursing ethics 19.6 (2012): 838-848.

11. Neff, K. Self Compassion. 2015. Available from: http://self-compassion.org/.

12. Heffernan M, Quinn M, McNulty R, Fitzpatrick J. Self-compassion and emotional intelligence in nurses. Int J Nurs Pract. 2010;16:366-73.

13. Freshwater $D$, Stickley $T$. The heart of the art: emotional intelligence in nurse education. Nurs Inq. 2004;11(2):91-8.

14. Moon T. Emotional intelligence correlates of the four-factor model of cultural intelligence. J Manag Psychol. 2010;25(8):866-10

15. Ang S, Dyne L Van, Koh S K. Personality correlates of the four factor model of cultural intelligence. Group and organization management. 2006:31:100-123.

16. Royal College of Nursing. Raising concerns: a guide for RCN members. 2015. Available at: http://www.rcn.org.uk/support/raising_concerns.

17. Bulmer Smith K, Profetto-McGrath J, Cummings G. Emotional intelligence and nursing: an integrative literature review. Int J Nurs Stud. 2009:46(12):1624-36.

18. Smith S, Gentleman M, Loads D, Pullin S. An exploration of a restorative space: a creative approach to reflection for nurse lecturer's focused on experiences of compassion in the workplace. Nurse Educ Today. 2014; 34:1225-31.

19. Francis R. Report of the Mid Staffordshire NHS Foundation Trust Public Inquiry Executive Summary. London: The Stationary Office; 2013.

20. Ballat J, Campling P. Intelligent Kindness Reforming the culture of healthcare. London: Royal College of Psychiatrists Publications; 2011.

21. Department of Health. Compassion in Practice: Nursing, Midwifery and Care Staff Our Vision and Strategy. 2012. Available form, http://www.england.nhs. uk/wpcontent/uploads/2012/12/compassion-in-practice.pdf.

22. Hugues $K$, Hood L. Teaching methods and an outcome tool for measuring cultural sensitivity in undergraduate nursing students. J Transcult Nurs. 2007; 18(1):57-62.

23. Nolan M, Davies S, Brown J, Keady J, Nolan J. Beyond 'person-centred' care: a new vision for gerontological nursing. J Clin Nurs. 2004;13(1):45-53.

24. Nolan M, Davies S, Brown J, Nolan J, Keady J. The Senses Framework: Improving Care for Older People Through a Relationship-Centred Approach, Getting Research Into Practice Series. Sheffield: University of Sheffield; 2006.

25. Coetzee $\mathrm{S}, \mathrm{Klopper} \mathrm{H}$. Compassion fatigue within nursing practice: a concept analysis. Nurs Health Sci. 2010;12:235-43.

26. Gentry J. Compassion fatigue: a crucible of transformation. J Trauma Pract. 2002;1(3/4):37-61.

27. Brown C. Experiential learning and compassionate care. In: Shea S, Wynyard $\mathrm{R}$, Lionis C, editors. Providing compassionate healthcare: Challenges in policy and practice. Oxon: Routledge; 2014. p. 54-67.

28. Smith S, Dewar B, Pullin S, Tocher R. Relationship centred outcomes focused on compassionate care for older people within in-patient care settings. Int J Older People Nursing. 2010;5:128-36. doi:10.1111/j.1748-3743.2010.00224.x.

29. Paley J. Social psychology and the compassion deficit. Nurse Educ Today. 2013:33:1451-2.

30. Timmins F, de Vries M. Nurses are not bystanders: a response to Paley. Nurse Educ Today. 2014;34(10):1269-71.

31. Iles V, Vaughan Smith J. Working in health care could be one of the most satisfying jobs in the world - why doesn't it feel like that? Paper from the Take it to the Limit' Learning Set. 2009. Available at: http://www.reallylearning. com/Current_Projects/Learning_Sets/take-it-to-the-limit-report2.pdf. 